\title{
The Importance of Interceptive Orthodontic Treatment
}

\author{
Ana Nancy Silberstein ${ }^{1,2, *}$ \\ ${ }^{1}$ World Federation of Orthodontists, Mexico \\ ${ }^{2}$ Asociación Mexicana de Ortodoncia, Mexico
}

*Corresponding author: Ana Nancy Silberstein, World Federation of Orthodontists, Mexico, Tel: 525555209651; E-mail: a_silberstein@hotmail.com

Received: 01 Apr, 2021 | Accepted: 12 Apr, 2021 | Published: 20 Apr, 2021

Citation: Silberstein AN (2021) The Importance of Interceptive Orthodontic Treatment. Int J Dent Oral Health 7(3): dx.doi.org/10.16966/23787090.361

Copyright: @2021 Silberstein AN. This is an open-access article distributed under the terms of the Creative Commons Attribution License, which permits unrestricted use, distribution, and reproduction in any medium, provided the original author and source are credited.

$60 \%$ of the children's facial development is completed by the age of 8 and $90 \%$ of the facial development is completed by the age of 12. This means that the earlier you visit an orthodontist, the better opportunity to detect and correct any growth and development problems that may be present.

The growth of the oral-facial region depends mainly on 2 factors: genetically and environmental.

There are several reasons which can cause an unequal growth of a child's face, and this imbalance may affect a child's health and appearance. A normal balanced face is the result of the sum of the individual growth of each bone, the muscle functioning, proper nutrition and the ability to breathe normally.

The World Health Organization recommends breastfeeding until 2 years old because of the benefits of breast milk, but when the baby nurses, it is also swallowing and breathing, so the changing pressure and the jaw motion helps the facial development.

Another important function is breathing properly. One of the most common abnormalities in a child's facial growth and development is caused by a compromised airway.

The inability to breathe properly through the nose results in breathing through the mouth. This sets up a chain of events that may severely impact the child's health, facial features to develop, and future child appearance.

The effects of a compromised airway on the growth of a child can be revealed in several ways: (1) The tongue often positions itself in the lower jaw, which can change the growth of the lower jaw in a vertical direction.

(2) As the nasal breathing is compromised, the upper jaw, nasal bones and cheekbones fail to develop normally because the airflow through the nose is absent.

Most of the common signs and symptoms are:

1. Lips apart
2. Gingivitis

3. Dark circles beneath the eyes

4. Change in head posture

5. Chronic sinus and throat problems

6. Snoring

7. Grinding teeth during sleep

8. Sleep difficulties

9. Ear infections

Obstruction of the airway can be caused by

- $\quad$ Enlarged adenoids

- $\quad$ Enlarged tonsils

- Nasal obstruction

- $\quad$ Allergies

- Chronic sinus infections

An Orthodontist can help develop a plan to correct the factors of facial growth abnormalities and restore normal growth. Help control bad habits such as thumb sucking, unilateral chewing, masticatory performance and frenulum size.

When corrected early in a child's life, facial growth can go back to harmony and balance, which impacts the beauty of a child and later as an adult.

A good diagnosis is important to develop a treatment plan, including other specialties.

There are many orthodontic procedures to achieve our goal. One of them is Invisalign First, which is designed to treat from simple to complex malocclusions.

The Invisalign technology helps to address the treatment in 3 dimensions. This treatment can be ideal for children 6-10 years old. 
You can achieve dental arch expansion in a very predictable way. The Smart Stage technology is applied by producing contacts and forces that support the teeth movement needed for the arch expansion.

You can decide between the simultaneous expansion of the arches or, to provide more anchorage and more targeted force to each segment of the arch. Molars start moving first, followed by simultaneous expansion of canines and posterior teeth, and the alignment of the incisors starts from the beginning of the treatment.

The Invisalign system has a compensation algorithm for the eruption of the permanent dentition. This gives us the possibility to treat patients in early to late mixed dentition.
Invisalign's first treatment is designed to accomplish molar rotation, molar distal movement and mandibular advancement.

Movements are biological and predictable and can be done either unilaterally or bilaterally, depending on the case.

The inclusion criteria for Invisalign First is to have permanent first molars, at least one primary molar in each arch side, and 3/4 of the crowns of 2 permanent incisors (upper and lower). Alternatively, to have strong primary incisors.

Invisalign's first clear aligner treatment is designed for predictable results and a positive experience while addressing the unique needs of growing children. 